\title{
Validation of the radiation pattern of the Middle Atmosphere Alomar Radar System (MAARSY)
}

\author{
T. Renkwitz, W. Singer, R. Latteck, G. Stober, and M. Rapp \\ Leibniz Institute of Atmospheric Physics at the Rostock University, Schloss-Str. 6, 18225 Kühlungsborn, Germany \\ Correspondence to: T. Renkwitz (renkwitz@iap-kborn.de)
}

\begin{abstract}
In 2009/2010 the Leibniz-Institute of Atmospheric Physics (IAP) installed a new powerful VHF radar on the island Andøya in Northern Norway $\left(69.30^{\circ} \mathrm{N}, 16.04^{\circ} \mathrm{E}\right)$. The Middle Atmosphere Alomar Radar System (MAARSY) allows studies with high spatial and temporal resolution in the troposphere/lower stratosphere and in the mesosphere/lower thermosphere of the Arctic atmosphere. The monostatic radar is operated at $53.5 \mathrm{MHz}$ with an active phased array antenna consisting of 433 Yagi antennas. Each individual antenna is connected to its own transceiver with independent phase control and a scalable power output of up to $2 \mathrm{~kW}$, which implies high flexibility of beam forming and beam steering. During the design phase of MAARSY several model studies have been carried out in order to estimate the radiation pattern for various combinations of beam forming and steering. However, parameters like mutual coupling, active impedance and ground parameters have an impact on the radiation pattern, but can hardly be measured. Hence, experiments need to be designed to verify the model results. For this purpose, the radar has occasionally been used in passive mode, monitoring the noise power received from both distinct cosmic noise sources like e.g. Cassiopeia $\mathrm{A}$ and Cygnus $\mathrm{A}$, and the diffuse cosmic background noise. The analysis of the collected dataset enables us to verify beam forming and steering attempts. These results document the current status of the radar during its development and provide valuable information for further improvement.
\end{abstract}

\section{Introduction}

In this manuscript we present a recent study with the main aim to validate the receiving system of the MAARSY VHF radar by observing cosmic radio noise. The architecture of the radar has been introduced in detail in Latteck et al. (2012). During the design phase of this radar, the radiation pattern of the active phased array antenna has been exten- sively simulated using the Numerical Electromagnetic Code (NEC 4.1). For the simulation of various combinations of beam forming and beam pointing, realistic ground parameters have been included. Nevertheless the radiation pattern may be affected by e.g. mutual coupling of the antennas and active impedances. This implies the necessity of experimental methods to verify the model results.

Non-thermal cosmic radio emissions on frequencies below $100 \mathrm{MHz}$ are mainly generated by synchrotron radiation of cosmic ray electrons and vary for each distinct point in the sky. In the 1930s the first extra-terrestrial radio emissions were detected and first intensity maps were created in the 1940s. Since then, radio astronomers accomplished several surveys using a wide range of the radio frequency spectrum to detect and study various sources of cosmic radio noise.

To obtain accurate measurements with atmospheric VHF radar systems it is vital to know the beam pointing direction and the beam shape itself as precisely as possible. During the last few decades observations of cosmic noise emissions have been carried out to calibrate various atmospheric radar systems (see e.g. Czechowsky et al., 1984; Palmer et al., 1996; Zecha, 1999; Campistron et al., 2001; Carey-Smith et al., 2003; Fukao et al., 2003; Swarnalingam et al., 2009a,b; Renkwitz et al., 2011). The purpose of these studies has been e.g. to derive the apparent noise contribution for a given sidereal time at the receiver system, to verify the beam pointing of the antenna array, or to estimate the beam pattern itself as a process of calibration. Additionally, cosmic noise observations allow the verification of the system sensitivity. Typical cosmic noise sources which have previously been studied for the above mentioned purposes are: e.g. Cassiopeia A (RA: 23 h 23.4 m, Dec: $+58^{\circ} 48.9^{\prime}$ ), Cygnus A (RA: 19 h 59.473 m, Dec: $\left.+40^{\circ} 44.035^{\prime}\right)$ on the Northern Hemisphere and Sagittarius A (RA: $17 \mathrm{~h} 43.25 \mathrm{~m}$, Dec: $-28^{\circ} 52^{\prime}$ ) on the Southern Hemisphere. In the following sections we describe the methods used to verify the beam pointing direction of MAARSY and to estimate the apparent beam width. Afterwards, we 


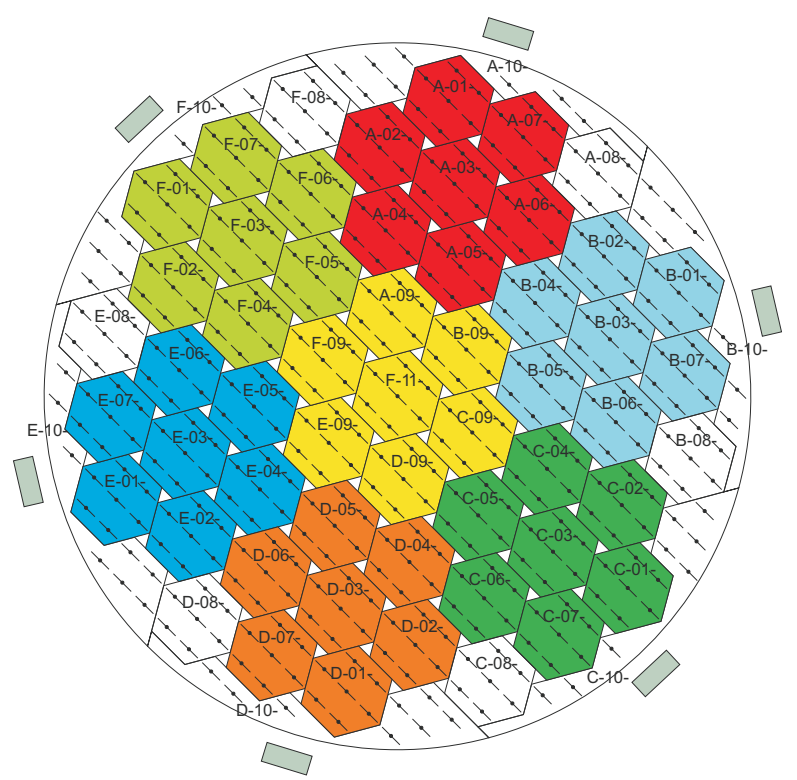

Fig. 1. Sketch of the MAARSY VHF radar antenna array. The seven anemone antenna groups used in this manuscript are marked with colors.

analyze the observed intensity of the two major cosmic noise sources to evaluate the gain degradation while pointing the beam far off-broadside. Additionally we will present a comparison of our observations with a global sky model which is based on several cosmic noise surveys.

\section{Experiment description}

With the flexible beam steering of the MAARSY radar, we are able to run passive scans within a cone of at least $30^{\circ}$ offzenith angle. Simulations show that within this zenith angle range MAARSY is free of grating lobes. During these passive scans the transmitter has been switched off to prevent any contamination of the data set. This contamination may appear within the system itself e.g. due to crosstalk in the transmit/receive-switches or by unfortunate range settings and, therefore, the reception of back scatter of the transmitted signal by any near or distant targets.

The general representation of total noise power $P_{\text {tot }}$ is composed of the external and internal contribution of noise power and is given by

$P_{\text {tot }}=k \cdot B \cdot\left(T_{\text {sky }}+T_{\text {rec }}\right)$

where $k$ is the Boltzmann constant, $B$ the observation bandwidth, $T_{\text {sky }}$ and $T_{\text {rec }}$ are the equivalent absolute noise temperatures of the observed sky and the receiver, respectively. Following this equation, $T_{\mathrm{rec}}$ limits the sensitivity of the radar, which should not change within the experiments. As the bandwidth is also kept constant, the incident noise power is controlled by the sky temperature captured by the radar antenna array.

In general, the most critical and essential part of all sky noise observations is the estimation of the Quiet Day Curve (QDC). The actually detected power may be contaminated by additional noise power of various origins, e.g. the influence of the highly variable sun has to be precluded. The sun may introduce additional noise power e.g. when passing through the antenna main beam or any side lobe of significant intensity or results in decreased detected incident power due absorption as a consequence of increased electron density in the D-region. Furthermore additional external artificial sources inflicting the observations have to be excluded prior to any subsequent analysis.

Various scan experiments have been performed during the different expansion stages of the radar to analyse the actual performance. Most of the cosmic radio sources are located at less than $70^{\circ}$ declination and culminate exactly south of the radar. Therefore, the experiments have been carried out to scan in southern directions $\left(\phi=180^{\circ}\right)$ at zenith angles of $\theta=0-34^{\circ}$ with an angular resolution of $1^{\circ}$. Due to the sensitivity, temporal and angular resolution the radar should be able to receive very distinct sources of radio emissions. During earlier experiments we were already able to detect the two strongest radio sources on the Northern Hemisphere, namely Cassiopeia $\mathrm{A}$ as a supernova remnant and Cygnus A as a radio galaxy. During November and December 2011 new experiments with almost the full aperture of the radar have been performed. These cosmic noise experiments were sequenced with other experiments carried out during that time, therefore the temporal resolution of the experiments analyzed in this manuscript is approximately $5 \mathrm{~min}$, while the data gaps have been interpolated for the analysis where necessary. The collected data set has been analyzed for a configuration, where we used 343 of the 433 antenna array elements, related to seven "anemone" antenna substructure, which are marked in Fig. 1. For every anemone an individual intermediate frequency signal is transferred to the 16-channel base band receiver and are subsequently sampled. Thus we combine the seven anemones in software to generate a beam with approximately $4^{\circ}$ beam width when pointing to zenith. During these experiments it was not possible to combine the complete array in software, therefore the subsequent analysis is restricted to 343 array elements, afterwards referred to as MAARSY343. The radiation pattern for the MAARSY antenna array using 7 anemones pointing to the radio sources Cassiopeia A and Cygnus A are depicted in Fig. 2.

For the subsequent analysis we exclusively used data from 1-2 December as during that time the geomagnetic activity and any additional interference to the receiver system appeared to be very low and thus the data can be used without any further manipulation.

The raw data have been analyzed as follows. First, for each beam pointing direction we generated the combined beam by respectively integrating the in-phase and quadrature 

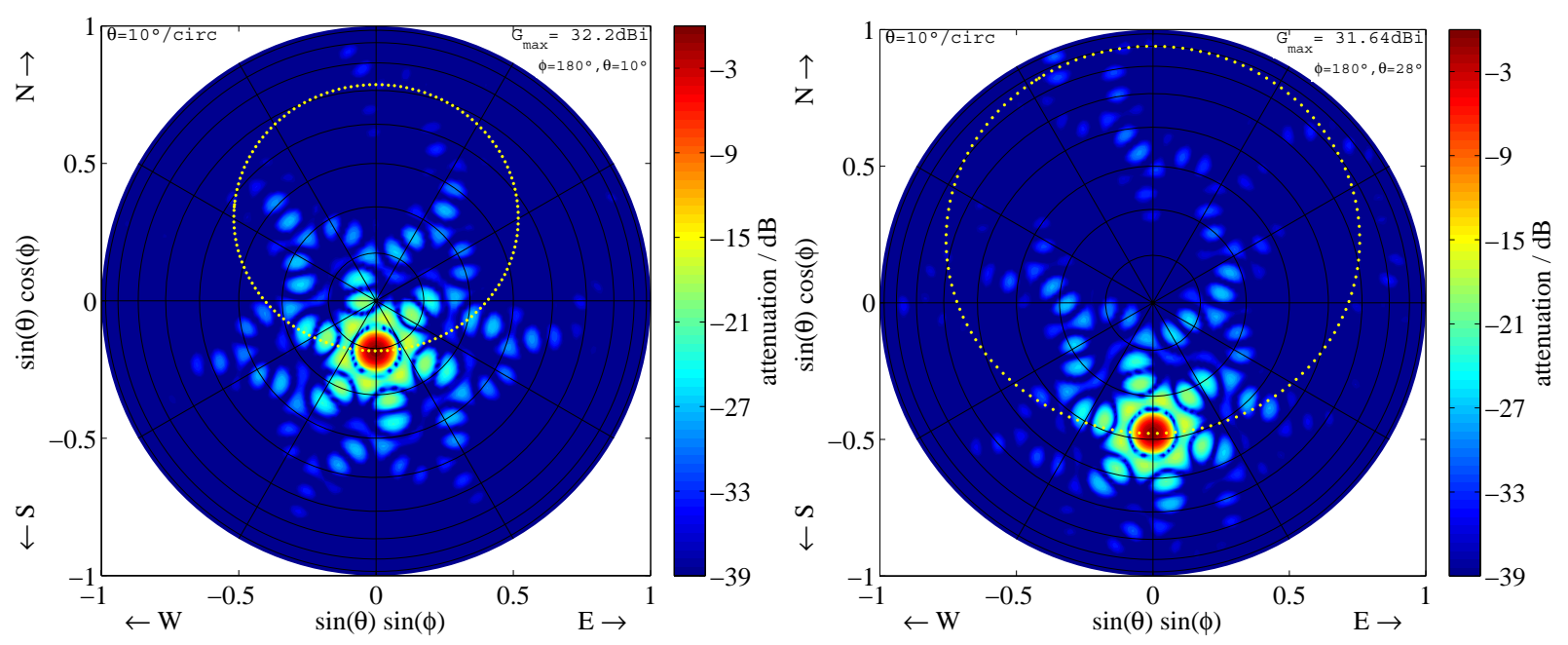

Fig. 2. Simulated radiation pattern of the MAARSY antenna array in top view, using 343 array elements while pointing to the radio sources Cassiopeia A $\left(\phi=180^{\circ}, \theta=10.5^{\circ}\right.$ - left side) and Cygnus A $\left(\phi=180^{\circ}, \theta=27.5^{\circ}\right.$ - right side). The corresponding trajectories of both radio sources are depicted with dotted circles. The individual radiation pattern are depicted relative to the maximum gain with an overlay of equidistant rings of each $10^{\circ}$ zenith angle. For these simulations the Numerical Electromagnetics Code (NEC) with the Norton-Sommerfeld approximation was used implicating the mutual coupling of antennas and the influence of imperfect ground.

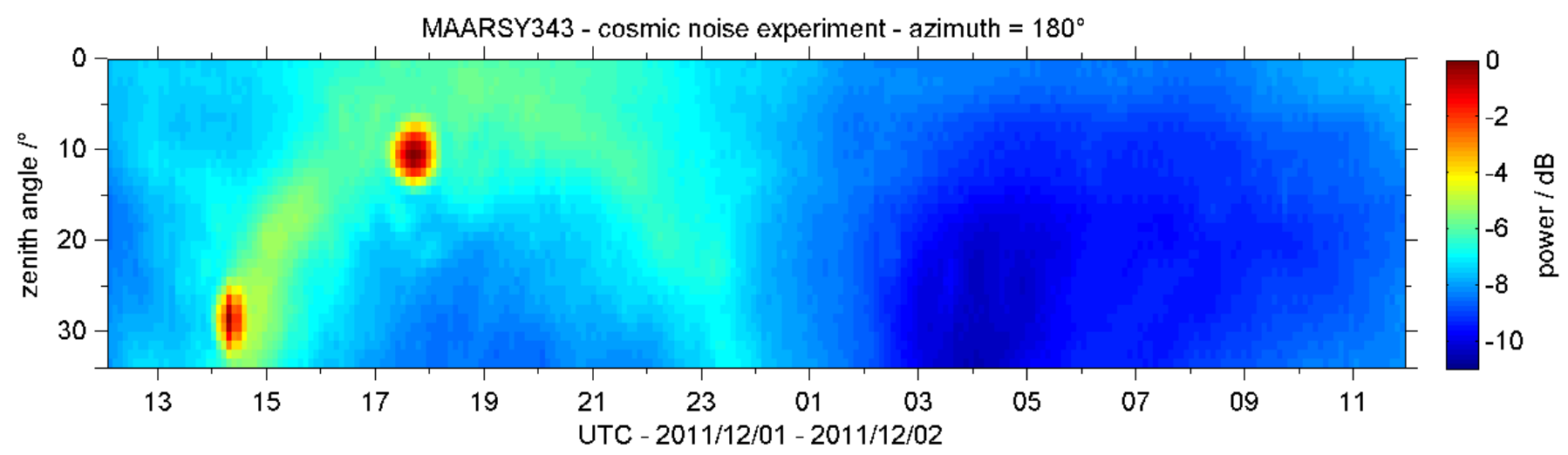

Fig. 3. Distribution of incident noise power over time and zenith angle $\theta$ for a scan within $\phi=180^{\circ}, \theta=0-34^{\circ}$. The highlighted point-like radio sources are Cassiopeia $\mathrm{A}\left(\theta=10.5^{\circ}\right)$ and Cygnus $\mathrm{A}\left(\theta=27.5^{\circ}\right)$.

components of the time series of the seven anemone signals. Subsequently we subtracted the DC-part (mean of the inphase and quadrature component, respectively) in every time series of the seven anemone signals and the aforehand generated combined beam. Afterwards, we derived the power of the received signal for each receiver channel by calculating the auto-correlation function of the corresponding time series. Applying this method we obtained a smoothed and robust time series for the whole observation.

The time series of this scan experiment is presented in Fig. 2, where the angular and temporal distribution of incident noise power is depicted. The two most pronounced sources Cygnus A and Cassiopeia A can be found at 14:15 UTC and 17:38 UTC, respectively. The detailed analysis of the apparent passage of the radio sources through the radar beam is described in the next section. Furthermore we see the accentuated spiral signature of the galaxy where also both radio sources are co-positioned. In total we see up to $10.5 \mathrm{~dB}$ dynamic range between the strongest source and the coldest point within the scan volume.

The aim of further experiments was to detect the radio source Taurus A (RA 05 h 34 m 31.97 s, Dec. +22 00'52.1"), which is connected to the supernova remnant Crab nebular. This radio source has been studied e.g. by Vinyajkin (2005) at a frequency of $151.5 \mathrm{MHz}$. Before, the IAP has never been able to observe this radio source as the earlier radar systems did not have the capability to steer the beam in the appropriate directions. However, even with the MAARSY radar Taurus A is well out of the designed and commonly used beam pointing cone. To monitor Taurus $\mathrm{A}$ the radar beam 


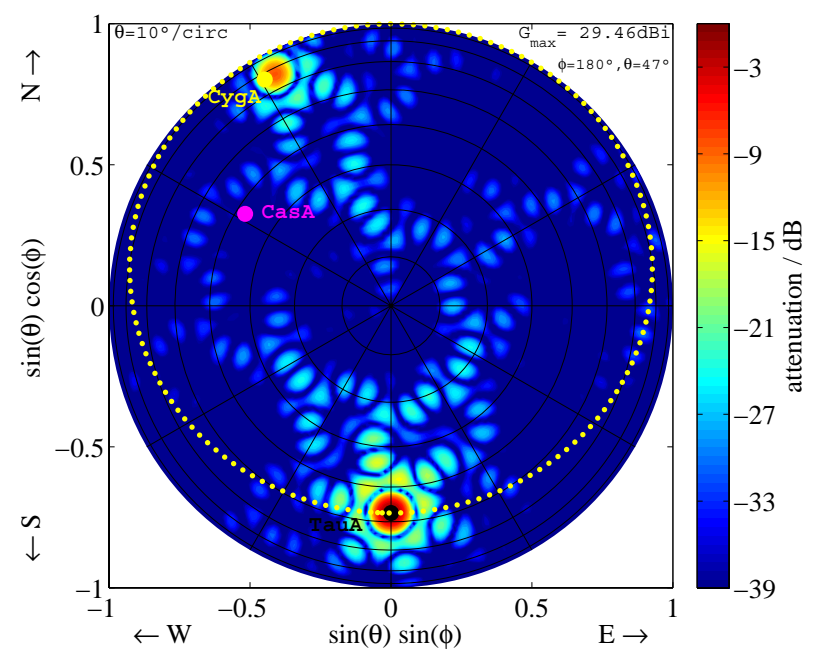

Fig. 4. Simulated radiation pattern of MAARSY343 while pointing to the radio source Taurus A $\left(\phi=180^{\circ}, \theta=47.3^{\circ}\right)$, superimposed by the current positions of Cygnus A, Cassiopeia A. In the uppermost left part the overlap of Cygnus A and the radars grating lobe can be seen.

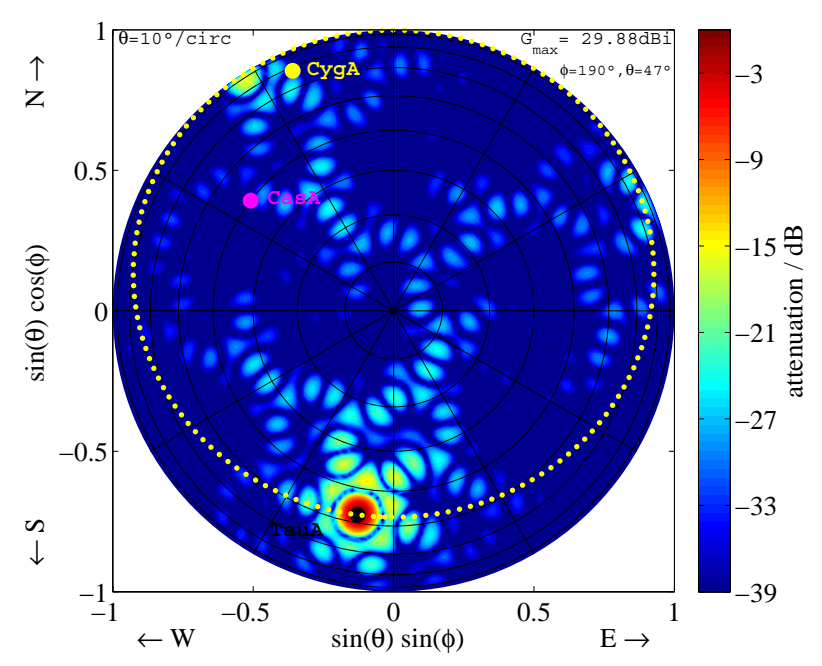

Fig. 5. Simulated radiation pattern of MAARSY343 pointing the radar beam to the radio source Taurus $\mathrm{A}$ at $\phi=190^{\circ}, \theta=47.7^{\circ}$, superimposed by the current positions of Cygnus A, Cassiopeia A. For this beam steering configuration the grating lobe is significantly separated from the current position of Cygnus A.

has to be pointed to at least $47.3^{\circ}$ zenith angle (see Fig. 4), which is the culmination of this radio source at the radars location. Obviously a scan within these high zenith angle values inherently generates grating lobes for our antenna array, which can also be seen in Fig. 4, where the main beam is generated at azimuth $\phi=180^{\circ}$ and zenith angle $\theta=47.5^{\circ}$. In the first experiment we were already able to detect the signature of Taurus A, however in this configuration, the grating lobe is close to overlap with the current position of Cygnus

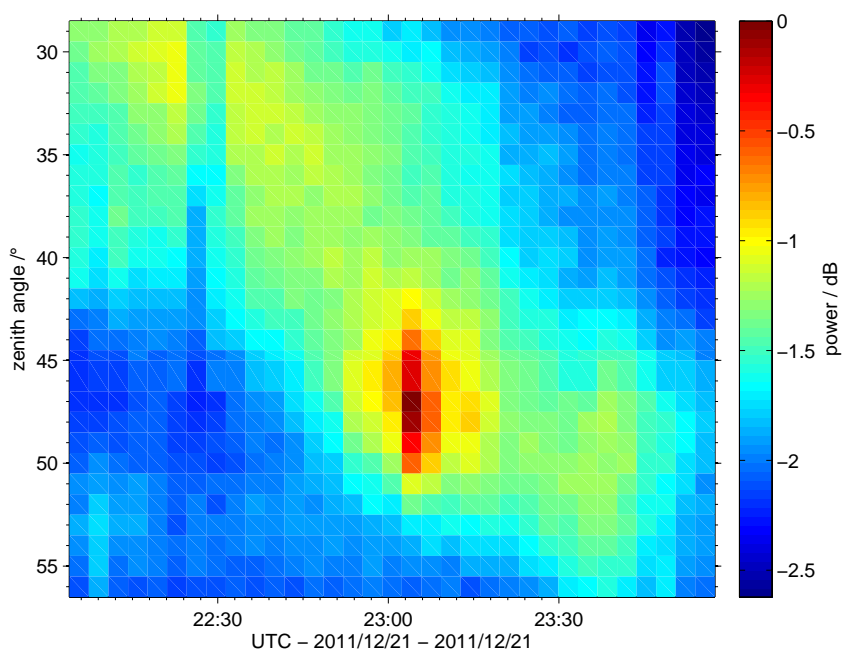

Fig. 6. Distribution of incident noise power for a scan within $\phi=190^{\circ}, \theta=28.5-56.5^{\circ}$. The maximum intensity is related to the radio source Taurus $\mathrm{A}\left(\theta=47.7^{\circ}\right)$, which is co-located to the galaxy (visible in yellow/green contour).

A $\left(\phi=330.8^{\circ}, \theta=67.8^{\circ}\right)$. To eliminate this potential contamination of received noise power via the grating lobe another experiment was defined. Within the new experiment Taurus A has been monitored having the radar beam pointing to $190^{\circ}$ azimuth instead of direct south, where we only lose about $0.25^{\circ}$ zenith angle to the radio source, but we gain about $20^{\circ}$ angular separation between the grating lobe and Cygnus $\mathrm{A}$ as at the same time the grating lobe is also generated in a different direction. This configuration is shown in Fig. 5, where the the contribution of Cygnus A to the received noise power should be attenuated by more than $20 \mathrm{~dB}$. During this experiment we could once again detect Taurus A (Fig. 6) and with this also verify the beam pointing capability of the MAARSY radar and estimate the accuracy to $1^{\circ}$ even for such high zenith angles. Furthermore this experiment demonstrates the potential use of the radar even outside its designed beam pointing cone with carefully chosen experiment parameters.

\section{Validation of the radar beam position and beam width}

The goal of the earlier mentioned scan experiments was to gain information about the actual beam pointing of the radar and to derive the actual beam width. As the detected total noise power typically fluctuates from sample to sample, other means have to be used to identify the apparent time of passage of the cosmic radio source, than simply selecting the time of maximum power. First, we trace the zenith angle containing the maximum noise power $\left(\theta_{\mathrm{P} \max }\right)$ in the near of the calculated zenith angle for the trajectory of the individual cosmic noise source. To increase the temporal resolution we interpolate the time series for zenith angles 

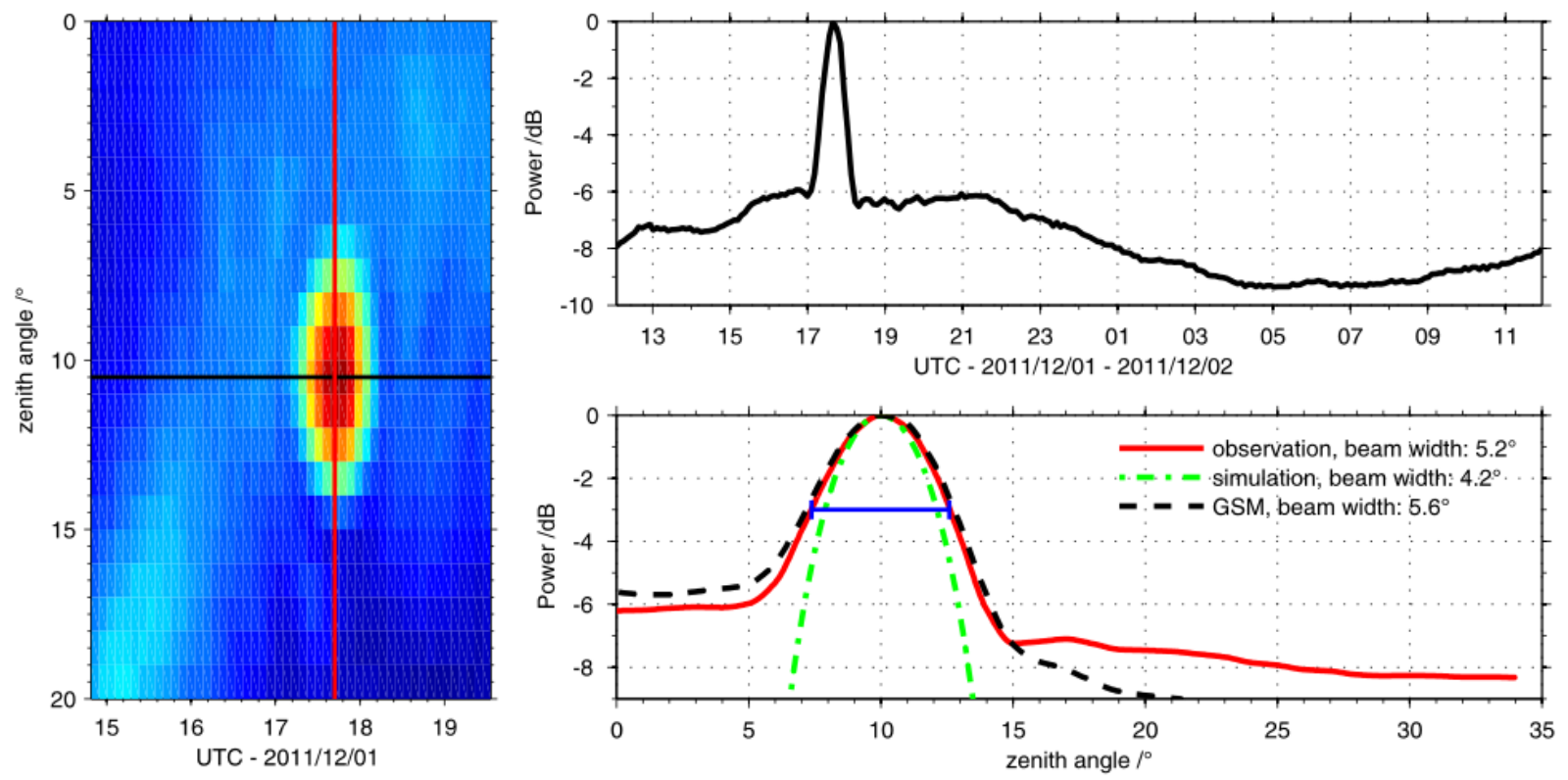

Fig. 7. Analysis of cosmic noise observations to estimate the apparent time of passage of Cassiopeia A. The left panel depicts the detected intensities during the experiment, where the subsequent cross sections are used to determine the apparent time of passage and beam width, which are marked by lines in the corresponding colors. The upper right panel shows the time series for a fixed zenith angle where the maximum intensity of the radio source has been detected. The lower right panel presents the cross section in zenith angle through the radio source. The estimated apparent beam width during this experiment, the simulated beam pattern and the model data are marked in red, green and black, respectively.

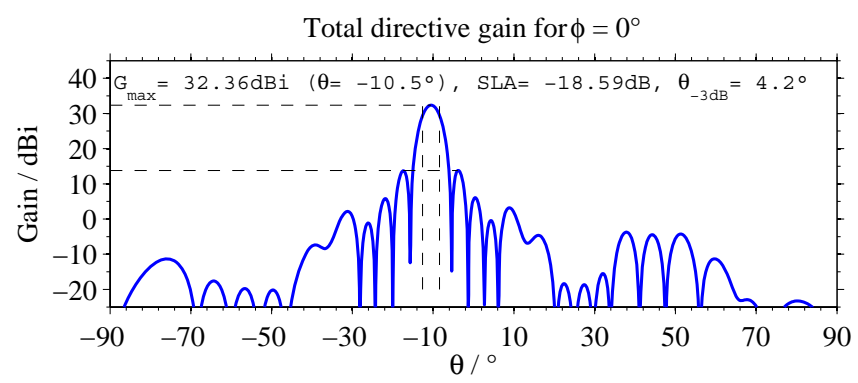

Fig. 8. Cross section of the simulated radiation pattern of MAARSY343, pointing to $\phi=180^{\circ}, \theta=10.5^{\circ}$ as a function of zenith angle $\theta$.

$\theta_{\mathrm{Pmax}} \pm 3^{\circ}$ with a cubic polynomial. Subsequently, we basically slide a scaled Gaussian function of adequate width over these equally-spaced interpolated time series. The best match of the interpolated time series with the shifted Gaussian function is derived by the method of least-squares. This sliding step of best match describes the apparent time of passage of the observed cosmic noise source and the analyzed zenith angles. This apparent time can now be compared to the calculated trajectory of the observed radio source, mainly giving information about the deviation of the radar beam in azimuth direction as the noise sources have been observed during their culmination. A similar method is subsequently used to derive the accuracy of beam pointing in zenith angle direction. The apparent beam width has been estimated for the points of half the noise power relative to the maximum. The result of this analysis for the radio source Cassiopeia A is shown in Fig. 7. For the experiments in December 2011 we were able to estimate the misalignment of the radar beam to be within $0.5^{\circ}$ in both azimuth and zenith angle, while we found a minimum beam width of $5.2^{\circ}$ in the direction of $\phi=180^{\circ}, \theta=10.5^{\circ}$ for the MAARSY343 configuration. In comparison, the beam width for this array configuration using 343 antennas as marked in Fig. 1 has been simulated to $4.2^{\circ}$ (see Fig. 8).

The apparent degradation of beam width is likely caused by the imperfect estimation of the apparent beam width by merely analyzing the $-3 \mathrm{~dB}$ points of the detected noise power. Therefore, we try to verify the beam width indirectly by using a global sky model of cosmic noise emissions, which will be used and introduced in more detail in the next section. To derive a representative curve we convolve the simulation of the complete radiation pattern including all side lobes with the global sky model data. For this, we used the radiation pattern of the antenna array pointing to $\theta=10^{\circ}$ and an approximation of it for the remaining scan directions. The result of this approach is depicted in Fig. 7 (dashed black line). The discrepancy above $15^{\circ}$ zenith angle is probably mainly related to the approximation used at these pointing directions. Nevertheless, our observations at and around the peak of Cassiopeia A match with the zenithal cross section from the global sky model. Hence the initially 
simulated radiation pattern has to be in agreement with the apparent radiation pattern that was existent during the observations.

Using the same data set we estimated the degradation of the antenna gain which appears while pointing the radar beam to greater off-zenith angles. The reason for this degradation is the influence of the radiation pattern of each individual array element to the array pattern. In our observations (see Fig. 2) we see about $0.3 \mathrm{~dB}$ less noise power from Cygnus A than from Cassiopeia A. The degradation of the antenna gain tilting the radar beam from Cassiopeia $\mathrm{A}$ $\left(\theta=10.5^{\circ}\right)$ down to Cygnus A $\left(\theta=27.5^{\circ}\right)$ have been simulated to be about $0.84 \mathrm{~dB}$, which results in a difference in absolute noise power of $0.54 \mathrm{~dB}$. Adopting the flux reference of ITU (2000) for our observation frequency the flux ratio between Cygnus A and Cassiopeia A is about $0.55 \mathrm{~dB}$. It should be noted that the emissions of Cassiopeia $\mathrm{A}$ are fading with a frequency dependent rate of 0.5 to $1 \%$ per year (see e.g. Bridle, 1967; Agafonov, 1996; Riahi, 2002). Uyanıker et al. (2004) studied e.g. flux densities of Cygnus A and it's detailed structure on e.g. $408 \mathrm{MHz}$. Vinyajkin and Razin (2004) estimated for observations on a frequency of $151 \mathrm{MHz}$ in the year 2005 a flux density rate of Cygnus A/Cassiopeia A to be in the order of 1.05 to 1.1 , which equals $0.46 \mathrm{~dB}$. They also estimated the annual fading rate of Cassiopeia $\mathrm{A}$ for $151 \mathrm{MHz}$ to be in the order of $-(0.88 \pm 0.09 \%)$ per year, which gives for 2011/12 a flux rate of approximately 1.136 between Cygnus A and Cassiopeia A, which equals to $0.8 \mathrm{~dB}$.

Generally we have to recognize that our observations are in good agreement to other observations, provided that the frequency dependence of flux and fading rate is taken into account. Additionally, it is worth to note that the propagation path through the atmosphere to the radio sources Cassiopeia A and Cygnus A during their culmination differ. Furthermore it has to be taken into account that the polarisation of the cosmic radio sources Cassiopeia A and Cygnus A differ, which might lead to variations in the detected noise power, when observing them with a fixed linear polarized antenna array (see e.g. Baars et al., 1964; Boland et al., 1966; Mayer and Hollinger, 1968; Downs and Thomson, 1972).

\section{Comparison of a single day data set of observations with a global sky model}

In the earlier section we derived information about the quality of the beam pointing and the beam forming of the radar resulting e.g. in the estimation of the current beam width. For this purpose we analyzed the received incident noise power from two distinct radio sources. In this section we will now compare the time series of $24 \mathrm{~h}$, assuming the data is free of any additional atmospheric damping or interference, to a global sky model. Surveys of cosmic noise radiation have been performed by several radio astronomers. One of the first extensive surveys has been presented by Haslam et al.

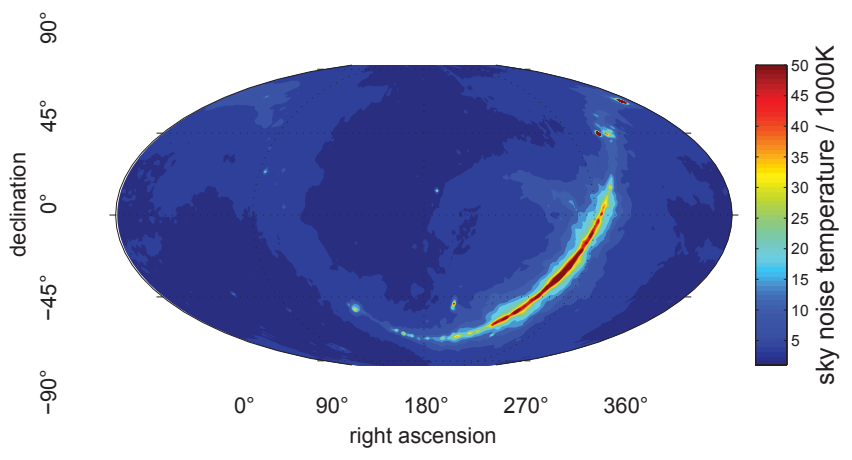

Fig. 9. de Oliveira-Costa et al. (2008) $53.5 \mathrm{MHz}$ radio image of our spiral galaxy transformed to a Mollweide projection in celestial coordinates. The resolution of the map is $1 \times 1^{\circ}$ in right ascension and declination. In the uppermost right part of the map the distinct contours of Cassiopeia A and Cygnus A are visible.

$(1974,1981)$ and (1982) for a frequency of $408 \mathrm{MHz}$, which is still used as an all sky reference nowadays. On even higher frequencies e.g. Reich et al. (1997) undertook detailed studies of cosmic noise emissions at approximately $1.4 \mathrm{GHz}$. For lower frequencies the earlier cosmic sky noise surveys e.g. Cane (1978) are based on observations with a $11^{\circ}$ wide beam, which is considerably large compared to MAARSY. The map of this survey appears smoothed due to the larger beam width and does not show the dynamic range. However, there have been narrow beam studies at $22 \mathrm{MHz}$ by Roger et al. (1999). Every of these studies implies valuable information for further comparisons, while the difference in the observation frequency has to be considered. Recently, de Oliveira-Costa et al. (2008) derived a model to create global sky noise temperature maps (GSM) for any user defined frequency in the range from $10 \mathrm{MHz}$ to $100 \mathrm{GHz}$. The model is based on the merging of the eleven most accurate sky noise surveys. At our frequency of interest, this results in a GSM with significantly increased resolution. The accuracy of the GSM is given to $1-10 \%$ depending on frequency and sky region. For our observation frequency the GSM is highly dependent on the $45 \mathrm{MHz}$ surveys of Maeda et al. (1999), which have been performed with the MU radar for the Northern declinations and on Roger et al. (1999) using the $22 \mathrm{MHz}$ DRAO radio telescope. The angular resolution and beam width in both surveys is better than the beam width we used for the observations with MAARSY. Hence the model data offers an appropriate angular resolution for further comparisons. A similar approach have been used by Stober et al. (2011) for the calibration of meteor radars. Applying this technique, we may gain additional information about the radiation pattern of the radar.

In the earlier section we almost exclusively examined the main beam in the analysis. However, all side lobes should be attenuated by more than $17 \mathrm{~dB}$, they nevertheless collect noise power from their individual heading. These side lobes 

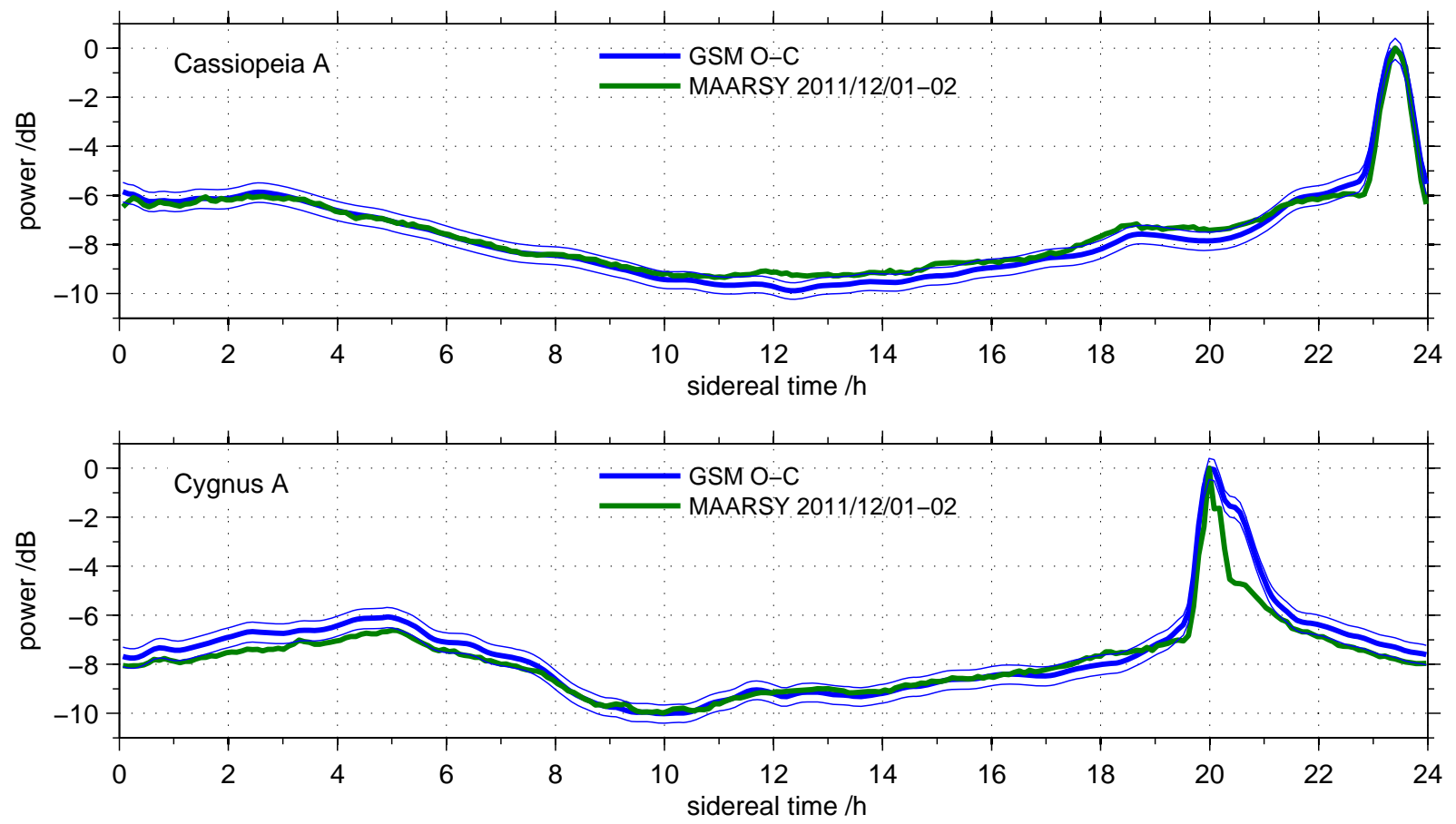

Fig. 10. Comparison of the GSM data (blue) to the observations with MAARSY (green) for Cassiopeia A (upper panel) and Cygnus A (lower panel), relative to the maximum intensity. The thin blue lines mark the accuracy interval of the GSM.

partially cover large areas of the sky and therefore contribute a significant amount of noise power. Thus the analysis should comprise the complete radiation pattern. For this purpose we convolve the simulated radiation pattern of the MAARSY antenna array with the GSM to generate a reliable Quiet Day Curve (QDC). To adapt the GSM data to the existing architecture of the MAARSY radar we introduced additional noise to the model to represent losses and the noise figure of the receiver. Therefore we include $1.3 \mathrm{~dB}$ loss for the main coaxial cable (converted to an equivalent noise figure $\mathrm{NF}_{\text {coax }}$ ) connecting the array elements to the receiver and a typical noise figure of $3.5 \mathrm{~dB}\left(\mathrm{NF}_{\mathrm{rec}}\right)$ for the receiver. The noise figure of a few receivers has been measured using a calibrated noise figure meter and the associated noise source. The measurement has been performed for the receiver input frequency of $54 \mathrm{MHz}$ through the front end and the down converter to the intermediate frequency of $11 \mathrm{MHz}$. Assuming that the front end and down converter of the receiver provide sufficient gain $(70 \mathrm{~dB}$ used in these experiments), the noise figure of these stages will dominate the subsequent stages (see Eq. 2).

$F_{\text {tot }}=F_{1}+\left(\frac{F_{2}-1}{g_{1}}\right)$

Where $F_{\text {tot }}$ is the total noise factor, the numbered indices denote the order of the amplifying stage while $F$ and $g$ are the corresponding linear noise factor and the gain, respectively.
Subsequently we convert the noise figure to the equivalent temperature $T_{\mathrm{e}}$ by using Eq. 3 , which results in $T_{\mathrm{e}}=585 \mathrm{~K}$.

$T_{\mathrm{e}}=T_{0} \cdot 10^{\left(\frac{\mathrm{NF}_{\mathrm{rec}}+\mathrm{NF}_{\mathrm{coax}}}{10}\right)}-1 \quad T_{0}=290 \mathrm{~K}$

The results of the comparison between our observations with MAARSY and the GSM data are presented for both radio sources Cassiopeia A and Cygnus A in Fig. 4 relative to their maximum power. The observation data is marked in green and the GSM data is depicted in blue including the $10 \%$ accuracy marked by thin blue lines. In general we clearly see a very good agreement between the observation and the model data.

In the comparison for both radio sources we see regions of almost perfect overlap, while for the rest the deviation remains less than $1 \mathrm{~dB}$. Interestingly, the overlap times of both QDCs are approximately the same, when e.g. the QDC for Cassiopeia A is shifted by $0.5 \mathrm{~dB}$, which implies a current overestimation of this source in the GSM. This may be related to the annual flux decrease of Cassiopeia A, which has been discussed in the prior section.

In the GSM data for Cygnus A is a clear second peak which appears about $30 \mathrm{~min}$ after the main peak of Cygnus A, which can also be seen in the observation data, however damped by about $3.5 \mathrm{~dB}$. This clear deviation may be related to the distinct spectral frequency distribution of the individual radio sources. As the GSM has been comprised by various observation data in the frequency spectrum from $10 \mathrm{MHz}$ to $100 \mathrm{GHz}$, maybe this distinct source is overestimated for 

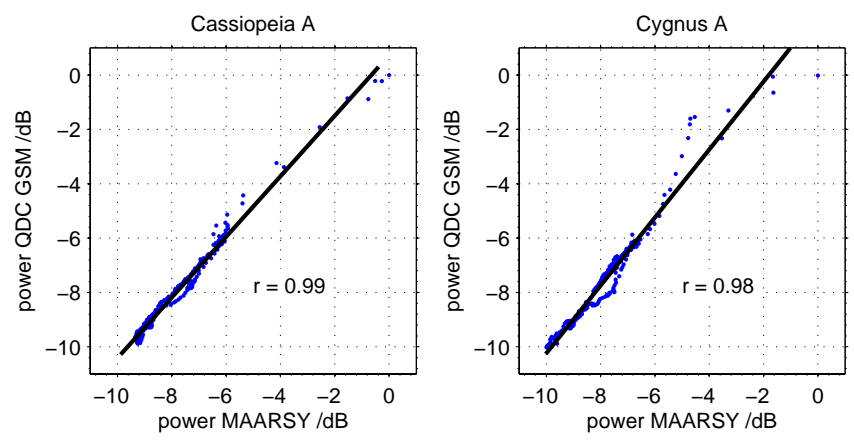

Fig. 11. Correlograms of the data comparison of GSM and the observations with MAARSY.

53.5 MHz. Nevertheless the correlation of both data sets is remarkably good with 0.99 and 0.98 for the two comparisons with Cassiopeia A and Cygnus A, respectively (see Fig. 4). As we have found such a good agreement to the independent GSM data set including the simulated radiation pattern, we are confident that the actual side lobes are not much stronger than we see in the simulations.

For the completeness, we renew the comparison of the flux ratio of Cygnus $\mathrm{A}$ and Cassiopeia $\mathrm{A}$, which have been analyzed in the end of the prior section. Using the GSM, we see a similar flux ratio and therefore a good agreement to our observations taking the accuracy of the model into account.

\section{Summary and conclusions}

In this manuscript, we analyzed experiments which have been performed to verify the beam pointing and beam forming of the MAARSY VHF radar. As the knowledge of these parameters is crucial for every experiment carried out with the radar, various methods were used to verify the extensive simulations, which were conducted during the design phase of the radar. For this purpose we monitored cosmic noise emissions and analyzed our observations of the two strongest radio sources Cassiopeia A and Cygnus A. Doing so, we verified the beam pointing and beam forming of the MAARSY radar and estimated the accuracy of beam pointing to be better than $0.5^{\circ}$ in azimuth and zenith angle. Subsequently we estimated the apparent beam width during these experiments. We found that the simple analysis of the detected noise power of the observed radio source is not sufficient to estimate the beam width. Instead, we used the simulation of our radiation pattern convolved with a global sky model of cosmic noise emissions which is composed of eleven most accurate sky noise surveys. Doing so, we found a good agreement with our observations, which underlines the validity of the simulated radiation pattern including the beam width used in these experiments. Furthermore we presented our first reliable observations of the radio source Taurus A, which is generally far out of the beam pointing cone of MAARSY. However, with carefully chosen experiment settings we were able to detect this well known radio source, which demonstrates the feasibility of such large zenith angle experiments. For Taurus A we estimated the accuracy of beam pointing to be better than $1^{\circ}$ in azimuth and zenith angle. Afterwards we compared the global sky model with our diurnal observation pointing the radar beam to the two major cosmic noise sources in the Northern Hemisphere. The corresponding quiet day curves of the model have been derived by including the complete radiation pattern for the individual beam pointing directions. In this comparison we found very high correlations of 0.98 and 0.99 for the observation of both major radio sources.

Overall, we are confident that our simulations of the radiation pattern of the MAARSY radar are consistent and reliable. Nevertheless, we intend to regularly include the noise observations to our experiments to assure the performance of the receiving part of our radar and to increase the accuracy of the estimated Quiet Day Curve. Additionally, we will perform more distinct cosmic noise observations with increased temporal resolution and expand the current observations to other azimuth angles to estimate the beam width also for other geographic directions. Especially, experiments using all array elements in a software combined beam will improve the angular resolution and thus also the dynamic range of these cosmic noise observations.

Acknowledgements. We like to express our gratefulness to the Andøya Rocket Range for the enduring support while building and operating the MAARSY radar. Furthermore, we highly appreciate the work of de Oliveira-Costa et al. (2008), which revealed to be a very valuable tool to perform passive radar calibrations. Topical Editor Matthias Förster thanks Gottfried Mann and Madhu Chandra for their help in evaluating this paper.

\section{References}

Agafonov, M. I.: Cassiopeia A flux density secular decrease and variations at metre wavelengths, Astron. Astrophys., 306, 578580, 1996.

Baars, J. W. M., Mezger, P. G., and Wendker, H.: The flux density values of standard sources used for antenna calibrations, Tech. rep., National Radio Astronomy Observatory, 1964.

Boland, J. W., Hollinger, J. P., Mayer, C. H., and McCullough, T. P.: Polarization of Cygnus A, Taurus A, and Cassiopeia A at 2.07 Centimeters, Astrophys. J., 144, 437-439, 1966.

Bridle, A. H.: Flux densities of Cassiopeia A and Cygnus A at 10.05 MHz, The Observatory, 87, 60-63, 1967.

Campistron, B., Despaux, G., Lothon, M., Klaus, V., Pointin, Y., and Mauprivez, M.: A partial $45 \mathrm{MHz}$ sky temperature map obtained from the observations of five ST radars, Ann. Geophys., 19, 863-871, doi:10.5194/angeo-19-863-2001, 2001.

Cane, H.: A $30 \mathrm{MHz}$ map of the whole sky, Aust. J. Phys., 31, 561565,1978 . 
Carey-Smith, T. K., McDonald, A. J., Baggaley, W. J., Bennett, R. G., Fraser, G. J., and Plank, G. E.: Antenna beam verification using cosmic noise, Proceedings of MST10 symposium, 2003.

Czechowsky, P., Schmidt, G., and Rüster, R.: The mobile SOUSY Doppler radar: Technical design and first results, Radio Sci., 19, 441-450, 1984.

de Oliveira-Costa, A., Tegmark, M., Gaensler, B. M., Jonas, J., Landecker, T. L., and Reich, P.: A Model of Diffuse Galactic Radio Emission from $10 \mathrm{MHz}$ to $100 \mathrm{GHz}$, Mon. Not. R. Astron. Soc., 338, 247-260, doi:10.1111/j.1365-2966.2008.13376.x, 2008.

Downs, G. S. and Thomson, A. R.: The distribution of linear polarization in Cassiopeia $\mathrm{A}$ at wavelengths of 9.8 and $11.1 \mathrm{~cm}$, Astron. J., 77, 120-133, 1972.

Fukao, S., Hashiguchi, H., Yamamoto, M., Tsuda, T., Nakamura, T., and Yamamoto, M. K.: Equatorial Atmosphere Radar (EAR): System description and first results, Radio Sci., 38, 1053-1070, doi:10.1029/2002RS002767, 2003.

Haslam, C. G. T., Wilson, W. E., Graham, D. A., and Hunt, G. C.: A further $408 \mathrm{MHz}$ survey of the northern sky, Astron. Astrophys., Supplement Series, 13, 359-394, 1974.

Haslam, C. G. T., Klein, U., Salter, C. J., Stoffel, H., Wilson, W. E., Cleary, M. N., Cooke, D. J., and Thomasson, P.: A 408 MHz allsky continuum survey. I - Observations at southern declinations and for the North Polar region, Astron. Astrophys., 100, 209 219, 1981

Haslam, C. G. T., Salter, C. J., Stoffel, H., and Wilson, W. E.: A $408 \mathrm{MHz}$ all-sky continuum survey. II - The atlas of contour maps, Astron. Astrophys., Supplement Series, 47, 1-143, 1982.

ITU: Determination of the G/T ratio for earth stations operating in the fixed-satellite service, Recommandation ITU-R S.733-2, Tech. rep., International Telecommunication Union, 2000.

Latteck, R., Singer, W., Rapp, M., Vandepeer, B., Renkwitz, T., Zecha, M., and Stober, G.: MAARSY: The new MST radar on Andøya - System description and first results, Radio Sci., 47, 1006-1024, doi:10.1029/2011RS004775, 2012.

Maeda, K., Alvarez, H., Aparic, J., May, J., and Reich, P.: A 45$\mathrm{MHz}$ continuum survey of the northern hemisphere, Astron. Astrophys., Supplement Series, 140, 145-154, 1999.

Mayer, C. H. and Hollinger, J. P.: Polarized brightness distribution over Cassiopeia A, the Crab Nebula, and Cygnus A at $1.55-\mathrm{cm}$ wavelength, Astrophys. J., 151, 53-63, 1968.
Palmer, R., Vangal, S., Larsen, M. F., Fukao, S., Nakamura, T., and Yamamoto, M.: Phase calibration of VHF spatial interferometry radars using stellar sources, Radio Sci., 31, 147-156, doi:10.1029/95RS02319, 1996.

Reich, P., Reich, W., and Fürst, E.: The Effelsberg $21 \mathrm{~cm}$ radio continuum survey of the Galactic plane between $l=95.5^{\circ}$ and $l=240^{\circ}$, Astron. Astrophys., Supplement Series, 126, 413-435, 1997.

Renkwitz, T., Singer, W., Latteck, R., and Rapp, M.: Multi beam observations of cosmic radio noise using a VHF radar with beam forming by a Butler matrix, Adv. Radio Sci., 9, 1-9, doi:10.5194/ ars-9-1-2011, 2011.

Riahi, N.: Cassiopeia A Flux Density Measured with APRAXOS, Tech. rep., Institute of Astronomy, ETH Zürich, 2002.

Roger, R. S., Costain, C. H., Landecker, T. L., and Swerdlyk, C. M.: The radio emission from the Galaxy at $22 \mathrm{MHz}$, Astron. Astrophys., Supplement Series, 137, 7-19, 1999.

Stober, G., Singer, W., and Jacobi, C.: Cosmic radio noise observations using a mid-latitude meteor radar, J. Atmos. Sol.-Terr. Phy., 73, 1069-1076, doi:10.1016/j.jastp.2010.07.018, 2011.

Swarnalingam, N., Hocking, W. K., and Argall, P. S.: Radar efficiency and the calculation of decade-long PMSE backscatter cross-section for the Resolute Bay VHF radar, Ann. Geophys., 27, 1643-1656, doi:10.5194/angeo-27-1643-2009, 2009a.

Swarnalingam, N., Hocking, W., Singer, W., and Latteck, R.: Calibrated measurements of PMSE strengths at three different locations observed with SKiYMET radars and narrow beam VHF radars, J. Atmos. Sol.-Terr. Phys., 71, 1807-1813, doi:10.1016/j. jastp.2009.06.014, 2009b.

Uyanıker, B., Reich, W., Yar, A., and Fürst, E.: Radio emission from the Cygnus Loop and its spectral characteristics, Astron. Astrophys., 426, 909-924, 2004.

Vinyajkin, E.: The Secular Decrease of the Crab Nebula at 927 and 151.5 MHz, Astrophysics, arXiv:astro-ph/0502033v1, 2005.

Vinyajkin, E. and Razin, V.: Secular Decrease and Random Variations of Cassiopeia A at 151.5 and $927 \mathrm{MHz}$, Astrophysics, arXiv:astro-ph/0412593v1, 2004.

Zecha, M.: Untersuchung der polaren sommerlichen Mesosphäre mit unterschiedlichen Radarverfahren im VHF-Bereich, Ph.D. thesis, University Rostock, 1999. 